Voix et Images

voixetimages

\title{
Édition, mouvements littéraires et paralittérature
}

\section{Bernard Andrès}

Volume 20, numéro 1 (58), automne 1994

Saint-Denys Garneau

URI : https://id.erudit.org/iderudit/201156ar

DOI : https://doi.org/10.7202/201156ar

Aller au sommaire du numéro

Éditeur(s)

Université du Québec à Montréal

ISSN

0318-9201 (imprimé)

1705-933X (numérique)

Découvrir la revue

Citer cet article

Andrès, B. (1994). Édition, mouvements littéraires et paralittérature. Voix et Images, 20(1), 237-245. https://doi.org/10.7202/201156ar d'utilisation que vous pouvez consulter en ligne.

https://apropos.erudit.org/fr/usagers/politique-dutilisation/ 
Recherche

\section{Édition, mouvements littéraires et paralittérature}

\section{Bernard Andrès, Université du Québec à Montréal}

Congrès d'écrivains, colloques d'universitaires, rencontres de théoriciens et de praticiens des lettres, autant de manifestations orales de l'art et du savoir qui finissent, quand les fonds le permettent, chez des éditeurs. Parmi les récentes publications du genre (la plupart chez Nuit blanche), j'ai retenu quelques titres relevant des questions d'esthétique, d'édition; d'écritures intimes, de mouvements littéraires et, surtout, de paralittérature.

À l'occasion du vingtième anniversaire de la Rencontre internationale des écrivains, Jean-Guy Pilon accueillait ses conférenciers de Sainte-Adèle sous le thème programmatique de "La beauté . Treize invités du Québec, mais aussi de France, de Belgique, du Liban, d'Italie, de Suède et du Brésil discouraient savamment de ce concept protéiforme qui nourrit l'objet artistique "de la Vénus antique aux néons du Pop Art". C'est à peu près dans ces termes que se trouve défini le colloque en question dans les actes parus à l'Hexagoné ${ }^{1}$. Du texte inaugural de Paul Zumthor pour qui l'expérience esthétique, savoir immense et inutile, déjoue le vrai comme le faux, à l'intervention dé Roger Grenier placée sous l'égide de Paul Valéry, chaque écrivain situe son propre rapport à la beauté comme forme, comme thème ou comme système d'opposition à des normes morales (bien, mal) ou esthétiques (beau, laid). Comme c'est souvent le cas dans ces rencon- 
tres dont le thème, très "accueillant ", laisse libre cours à l'imagination ou à la verve de chacun, les sensibilités, les approches nécessairement variables, frisent l'hétéroclite. Priment alors l'invention, l'astuce, l'humour avec lesquels le thème est abordé, sinon traité. Priment alors l'écriture, l'écrivain, l'esthète, l'esthétisme de certains propos, même. À l'esthéticien patenté d'aller chercher ailleurs, lui qui "fort laid, dans une salle incolore, parle de la beauté ${ }^{2}$.

Dans la mouvance des travaux du Groupe de recherche sur l'édition littéraire au Québec (GRELQ), Jacques Michon a réuni une série d'études dont la plupart ont fait l'objet d'un premier colloque en 1991. Elles concernent toutes la période stratégique des. années vingt et trente où une nouvelle génération d'éditeurs, d'animateurs et d'écrivains nationalistes négocient une forme d'autonomie entre le pouvoir clérical encore tout-puissant et les appareils d'État animés, eux, par des préoccupations surtout partisanes en matière culturelle. Grâce à l'intervention du pouvoir public, une certaine laicisation des entreprises de presse voit alors le jour, dont tireront profit des éditeurs comme Albert Lévesque. C'est ce vaste mouvement qu'étudie le livre préparé par Jacques Michon, L'Édition littêraire en quête d'autonomie ${ }^{3}$. Roman, poésie, essai philosophique et religieux témoignent alors de choix éditoriaux nouveaux et parfois risqués. Autour des maîtres d'œuvre Albert Lévesque et Albert Pelletier, de nouvelles maisons font leur entrée dans une modernité québécoise dont les analyses ici présentées retracent l'évolution (éditions Lévesque, du Totem ${ }^{4}$, etc.). C'est aussi le-rapport de l'écrivain à son texte et à son éditeur: Albert Laberge optant pour l'auto-édition (Paul Wycinski), Germaine Guèvremont et les libertés prises dans l'établissement de son Survenant (Yvan G. Lepage), Alfred Desrochers et l'"écurie" de la Librairie d'Action canadienne-française (Richard Giguère) ${ }^{5}$. Aux analyses de Suzanne Pouliot sur l'édition d'enfance et de jeunesse et d'Yvan Cloutier sur les éditions dominicaines du Lévrier, s'ajoute l'étude pénétrante de Michon sur Albert Lévesque, utilement complétée par un inédit de l'éditeur: Lévesque rectifiant en 1971 le deuxième tome des Mémoires de Lionel Groulx. Toute une correspondance est alors citée par Lévesque, qui nous éclaire sur les positions tranchées de l'un et l'autre animateurs littéraires. La figure d'Albert Lévesque en ressort d'autant mieux que le témoignage de sa fille Claire vient ensuite affiner le profil de l'homme et nous documenter en détail et comme à vif 
sur son étonnante carrière. Notons l'importance des lettres convoquées dans ces mises au point et dont le chercheur sait tirer parti dans son analyse des faits littéraires. Je signale à ce propos la parution récente du volume collectif La Lettre à la croisée de l'individuel et du social, sous la direction de Mireille Bossis ${ }^{6}$. Après une section sur l'émergence de la lettre comme genre aux XviI ${ }^{e}$ et Xvir ${ }^{\mathrm{e}}$ siècles, on y lit des études sur la diversification des pratiques aux $\mathrm{xIx}^{\mathrm{e}}$ et $\mathrm{xx}^{\mathrm{e}}$ siècles, avec notamment, des contributions québécoises de Manon Brunet sur la correspondance entre Casgrain et le marquis de Lévis, de Francis Parmentier sur Arthur Buies et de Roch Hurtubise sur les lettres d'amour au Québec entre 1860 et 1988. Ces mêmes auteurs avaient aussi contribué au volume dirigé par Manon Brunet et Serge Gagnon, Discours et Pratiques de l'intime. L'épistolaire y trouvait aussi sa place, mais aussi la lecture en Nouvelle-France (François Melançon), le charivari (René Hardy), la confession et le courrier du cöeur (Serge Gagnon) et divers travaux sur le journal intime ${ }^{7}$.

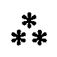

L'histoire littéraire est friande d'appellations commodes et de périodisations éprouvées (souvent ailleurs), qu'elle reprend à son compte, sans trop s'interroger sur leur pertinence bic et nunc. Il en va du classicisme comme du symbolisme ou du romantisme, mouvements qui recoupent en fait des réalités bien distinctes, d'un pays, d'une époque à l'autre. À l'heure où la réforme du français au Cegep force les professeurs à se situer dans ces repères, à les enseigner, à cerner les lettres québécoises dans la mouvance des chefs-d'œuvre français ${ }^{8}$, qu'en est-il du romantisme, par exemple? Comment en parler ici sans d'office établir une hiérarchie des productions, un palmarès des auteurs majeurs, mineurs ou "attardés"? C'est ce que se sont demandé les organisateurs du colloque "Le romantisme au Canada ${ }^{9}$ ". L'intuition de départ, formulée par Maurice Lemire, était que, loin de méconnaître ledit mouvement littéraire, les Canadiens ne l'ont pas pris en charge avec la même intensité qu'en France, et ce, parce qu'il correspondait moins à leur sensibilité ou à l'évolution de leur propre histoire nationale. Sauf chez Crémazie, Casgrain et Fréchette (mais avec un certain décalage), les Canadiens rejettent plutôt le romantisme, perçu comme une forme de décadence par rapport aux chefs-d'œuvre classiques. S'ils le pratiquent, c'est sur un mode bien à eux dont Lemire revendique le particularisme: "des circonstances différentes produisent nécessairement des résultats différents ". En outre, bien que 
prépondérant ${ }^{10}$, le modèle français n'est pas le seul à travailler l'imaginaire canadien (on se rappelle les innombrables références anglaises chez Aubert de Gaspé fils). Michel Pierssens soulève justement la question des multiples relais européens du romantisme et de la fluctuation du concept, de Chateaubriand à Mme de Staël; de Gothe à Sismondi ou Benjamin Constant ${ }^{11}$.

C'est ainsi que se construit un "paradigme européen." du romantisme, avec, dans la première section de ce volume, les contributions de Joseph Melançon sur le romantisme dans l'enseignement et d'ÉvaMarie Kröllẹr sur la filière allemande dans "l'encyclopédisme romantique au Québec". Les collaborateurs examinent ensuite les premières manifestations du mouvement littéraire, de 1815 (reprise marquée des relations culturelles avec la France), à la fameuse arrivée de La Capricieuse (1855): Claude Galarneau retrace cette évolution dans les migrations du personnel littéraire, alors que Kenneth Landry scrute le phénomène dans la circulation du livre à l'époque. La couleur particulière dú romantisme québécois fait l'objet d'une nouvelle section où Louis Rousseau souligne la part du renouveau religieux dans le romantisme québécois. Il s'intéresse plus particulièrement au prêtre de Saint-Hyancinthe, Joseph-Sabin Raymond, alors que Maurice Lemire interroge le retour qui s'effectue alors vers les écrits de la NouvelleFrance. À travers les initiatives de Jacques Viger, de Michel Bibaud, de Georges-Barthélemi Faribault et de Pierre-Jean de Sales Laterrière, il montre parfaitement la tension qui s'exerçait alors entre bibliographes et archivistes à propos de la constitution et de l'interprétation d'un corpus de référence. C'est que, parallèlement à cet effort de documentation sur le passé, une certaine conception de l'histoire et de la littérature se met en place: histoire et litterature ne naissent-elles pas de concert, autour de ce que Manon Brunet appelle "l'institutionnalisation du romantisme " au $\mathrm{xIx}^{\mathrm{e}}$ siècle? Casgrain assoit en effet les acquis de Garneau et toute une génération prend parti à la querelle sur le(s) romantisme(s). Si elle convient comme Manon Brunet du rôle de Casgrain dans cette "apothéose" du récit romantique (vers 1844-1845), Micheline Cambron demeure quant à elle fort critique à l'égard du bon abbé. Elle lui reproche un romantisme "de façade", dépourvu de "l'âme même du romantisme, c'est-à-dire l'ancrage dans une conception vectorielle de l'histoire où le présent serait le point du temps à partir duquel le progrès - et par ricochet toute la temporalité prendrait son sens" (p. 174). On le voit, parler du romantisme, c'est se situer soi-même dans l'idéologie du mouvement et porter un jugement sur ceux qui s'y inscrivirent, le commentèrent et, peut-être, l'enterrèrent. La vigueur des débats d'alors et d'aujourd'hui témoigne au moins 
du caractère crucial de ce mouvement littéraire. Sur ce plan, la dernière section du volume est assez explicite: qu'il s'agisse de roman (André Sénécal), de poésie (Paul Wyczynski), de légende (Fernand Roy et Lucie Robert), de théâtre (Jean Laflamme), d'écrits intimes (Laurent Mailhot) ou d'éloquence (Pierre Rajotte), le romantisme au Québec s'illustra dans à peu près tous les genres. D'où la nécessité d'en prendre acte et l'importance de ces actes.

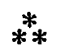

Dans l'esprit de cette relecture du passé littéraire et de certaines figures comme celle de' Sales Laterrière, je signale la réédition, par John Hare, d'un texte de François Baby datant de 1794: Le Canadien et sa femme ${ }^{12}$. Cet ouvrage de propagande nous replonge à l'époque des grands débats sur la langue et la politique, dans la dernière décennie du $\mathrm{xvIII}^{\mathrm{e}}$ siècle. Sur cette période décisive pour la formation d'une identité québécoise, il convient de lire l'autre récent ouvrage de John Hare, Aux origines du parlementarisme québécois ${ }^{13}$. Après avoir retracé le climat intellectuel au lendemain de l'Acte constitutionnel de 1791, l'auteur propose un copieux dossier documentaire qui rend enfin accessibles les archives de l'époque, avec des textes d'Alexandre Dumas (le nôtre!), de Pierre-Amable de Bonne, et moult exemples d'éloquence parlementaire (à faire pâlir tels de nos députés et actuels ministres). C'est dans cette mouvance discursive et idéologique que naît à nos yeux une certaine idée du littéraire: des "proto-scripteurs" se mettent à l'œuvre et, pour la première fois dans l'histoire des Canadiens, affrontent l'opinion publique. Ils s'exercent dans un nouvel espace conquis par l'imprimerie, les gazettes et les "magasins". Dans ces nouveaux circuits de discussion, clubs et loges maçonniques; 'c'est aux côtés des Jautard, Mesplet, 'Panet, Quesnel et du Calvet que prennent la plume La Corne, Mézière, de Messein, Sanguinet, Badeaux et d'autres dont le premier tome de La Vie littéraire a donné un bon aperçu ${ }^{14}$. C'est l'objet de toute une recherche à laquelle nous nous consacrons nous-même en publiant notamment les Cahiers de l'ALAQ. Dans Principes du littéraire au Québec, cette problématique est exposée, avec des contributions de René Beaudouin sur Pierre de Sales Laterrière, de Roger Le Moine sur la franc-maçonnerie et de Pierre Lespérance sur Luc Saint-Luc de La Corne ${ }^{15}$. Quant à Pierre-Jean de Sales Laterrière dont il était question plus haut, il s'agit du fils du médecin et mémorialiste Pierre de Sales Laterrière. Canadien installé à Londres et auteur d'un essai sur le Bas-Canada, il a aussi tenu le journal de ses 
voyages en Amérique du Nord et en Europe. Nous avons publié récemment un de ses inédits d'inspiration déjà romantique pour l'époque $(1815)^{16}$.

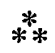

Parallèlement à ces travaux "classiques" d'histoire littéraire, d'autres recherches se poursuivent sur des périodes et des problématiques contemporaines, ou sur des corpus marginalisés par l'institution. Les études sur la littérature de grande consommation se portent bien, si l'on se fie notamment à l'annonce d'une nouvelle revue internationale Para $\bullet D o x a^{17}$, à la parution chez Nuit blanche d'un ouvrage sur la science-fiction et le fantastique, suivie par la naissance sous le même toit d'une nouvelle collection: "Études paralittéraires", dirigée par Norbert Spehner. L'ancien responsable de la collection "Paralittérature" au Préambule publie en effet coup sur coup deux volumes sur l'espionnage. L'un, de son cru, est un travail de collecte bibliographique: $\hat{E}$ crits sur le roman d'espionnage ${ }^{18}$. L'autre est un ouvrage d'analyse et de théorie du genre, que l'on doit à Paul Bleton (j'y reviendrai). Les Ailleurs imaginaires ${ }^{19}$ regroupe une série d'études livrées initialement en colloque, sur les rapports entre fantastique et science-fiction. Émanation du Groupe de recherche interdisciplinaire sur les fantastiques dans l'imaginaire québécois (GRILFIQ), cette rencontre vise à redéfinir les frontières entre deux régions voisines de la création littéraire. Pour les caractériser (trop schématiquement, bien sûr), ne pourrait-on dire que l'une nous émeut de l'intérieur (l'autre, l'étrange, l'extraordinaire est à la porte, gît en nous-même: fantastique), et l'autre nous entraîne aux limites de l'univers, d'autres univers (S.-F.). Avec tous les aménagements, déplacements et dépassements possibles auxquels se risquent, on l'entend bien, les spécialistes de ce volume. Comme le rappelle Simone Vierne, "on peut aussi jouer sur deux tableaux...". Alors que Roger Bozetto tente une archéologie de cet imaginaire, André Carpentier témoigne de sa propre expérience d'écriture aux confins des deux genres et Guy Bouchard aborde la question par le biais des utopies féministes. Élisabeth Vonarburg, pour sa part, se dédouble pour étudier chez elle-même (à la troisième personne), comme dans un plus vaste corpus, "la reproduction humaine individuelle comme motif-pivot de l'action". C'est aussi le corps, mais celui du monstre, qui se trouve "démonstré" dans l'étude de Patricia Willemin: de Jean Ray à Esther Rochon, en passant par Lewis, Ghelderode, Mary Shelley, Lovecraft et Bram Stocker, le tératogène fantastique est mis à nu. 
La réflexion, dans ce recueil d'études, emprunte les voies les plus variées: psychanalytique chez Renald Bérubé, socio-critique et anthropologique chez le regretté Jean Fabre, comparée chez Madeleine Borgomano et Antonio Risco, thématique chez Gilles Pellerin. Que l'approche soit externe (Jean-Marc Gouanvic, Rita Painchaud), ou interne, qu'elle insiste sur les contraintes génériques, éditoriales, institutionnelles ou historiques, ou qu'on s'en tienne au tissu textuel et à une forme d'immanence, la critique ne peut que constater le caractère fuyant de ces productions en rupture avec les codes réalistes de la représentation. Si Christian Vandendorpe risque une formalisation englobante des deux genres (tout en se défendant de figer l'objet dans sa grille), Michel Lord me semble bien cerner les points de rencontre entre ces deux univers discursifs:

[...] l'organisation du descriptif est aussi contraignante en fantastique qu'en SF, car il s'agit toujours de créer [...] l'illusion référentielle, de marquer avec force, comme une "chose "qui s'impose, la présence de l'impossible ou de l'improbable. Enfin, le type de questionnement sur le réel, l'irréel et le nouveau peut varier, mais, au fond, l'irrationnel n'est pas exclusif au fantastique, et le rationnel à la $\mathrm{SF}[\ldots]^{20}$.

Question d'écriture, question de lecture, aussi bien, comme le montre d'entrée de jeu Paul Bleton en s'interrogeant sur l'opération de catégorisation du genre.

Revenons donc avec ce chercheur à la question plus vaste de la paralittérature comme système. Bleton travaille depuis de nombreuses années sur le roman d'aventure, les polars et, plus généralement, sur la paralittérature, de la lecture sérielle à la B. D. Il vient de publier un fort volume sur un des cantons de cet univers : le roman d'espionnage $^{21}$. Sous le titre alléchant et mimétiquement proche des séries en question (Les Anges de Machiavel!), il nous offre une vaste réflexion sur les récits du genre publiés en France des débuts de la guerre froide à l'ère gorbatchévienne. S'expliquant sur les limites linguistiques du corpus, il fait justement remarquer que la production anglophone jouit d'une reconnaissance et d'un appareil critique déjà suffisamment fourni, en regard des travaux consacrés aux séries francophones. Notons toutefois qu'au plan de la reconnaissance institutionnelle, si le genre ne jouit pas encore parmi les littéraires d'un statut aussi "noble" que le roman policier, du moins bénéficie-t-il de la considération des subventionneurs (et donc des pairs) qui ont soutenu cette recherche (tout comme celles de Denis Saint-Jacques et de ses collègues de Laval sur le phénomène. IXE-13, les pratiques culturelles de grande consommation, ou celles de Julia Bettinotti sur le roman d'amour ${ }^{22}$ ). 
Le travail de Bleton constitue une mise au point rigoureuse et fort documentée sur le récit d'espionnage, du triple poịnt de vue de sa définition, de sa description et de la critique même du genre. Quels types de récits, quels topoïs y trouve-t-on à l'œuvre, en fonction de quelles traditions et contraintes éditoriales, et selon quel rapport à la société de référence? Comment ce genre compose-t-il avec l'Histoire et l'idéologie? Quel rôle y est-ịl dévolu au lecteur? Et comment la critique saisit-elle cet objet (ou échoue-t-elle dans sa saisie)? On le voit, loin de s'en tenir à une approche sociologique, Bleton recourt à l'analyse du discours, à une théorie de l'institution, à une sociocritique attentive aux questions formelles ${ }^{23}$ et à une sémiotique de la lecture. Ajoutons que l'essai lui-même se lit avec plaisir, compte tenu de la distance et de l'humour que l'auteur cultive à l'égard de son objet: tout ensemble critique et passionné, avec un bonheur dans l'expression peu coutumier chez les pairs (jusque dans les savoureux intertitres: "Le mauvais genre des espions de papier", "L'espion et son double", "Le report du guerrier: le sexe parangon", "L'envers vu des corps", et j'en passe). L'ouvrage fera date dans le domaine par la passion qui l'anime, mais aussi par un humour qui nous réconcilie presque avec le genre. N'oublions pas le second sous-titre de ces Anges: Froide fin et funestes moyens: les espions de papier dans la paralittérature française, du Rideau de fer à la chute du Mur...

Chut!

1. Louise Maheux-Forcier et Jean-Guy Pilon (dir.), La Beauté, l'Hexagone, 1993, 155 p.

2. Citation de Valéry donnée par Roger Grenier (p. 107).

3. Jacques Michon, L'Édition littéraire en quête d'autonomie: Albert Lévesque et son temps, Sainte-Foy, Les Presses de l'Université Laval, 1994, 216 p.

4. Voir l'article de Liette Bergeron sur les Éditions du Totem (p. 41-56) et son Catalogue de la Librairie d'Action canadienne-française et des Éditions Albert Lévesque (p. 165-200), ainsi que son mémoire de maitrise sur le sujet (Université de Sherbrooke, 1992).

5. Voir aussi le dossier que Richard Giguère avait consacré ici-même à Desrochers (Voix et Images, $\mathrm{n}^{\circ}$ 46, automne 1990).

6. Mireille Bossis (dir.), La Lettre à la croisée de l'individuel et du social, Paris, Kime, 1994, $254 \mathrm{p}$.

7. Ceux de Daphni Beaudoin, Hans Jürgen Lüsenbrik, Stéphane Stapinsky, Denise Lemieux et Gilles Houde, dans Manon Brunet et Serge Gagnon, Discours et Pratiques de l'intime, Québec, Institut québécois de recherche sur la culture, 1993, $269 \mathrm{p}$.

8. Pour réfléchir sur une base documentée à ces questions, je signale l'ouvrage collectif de Joseph Melançon, Clément Moisan, Max Roy et leurs collègues, La Littérature au cégep (1968-1978). Le statut de la littérature dans l'enseignement collégial, Québec, Nuit blanche, 1993, 420 p.

9. Voir Maurice Lemire (dir.), Le Romantisme au Canada, Québec, Nuit blanche, 1993, $342 \mathrm{p}$. 
10. Dans le même volume, David $M$. Hayne dresse la liste des écrivains français qui hantent la production québécoise de 1820 à 1845 .

11. Claude Foucart, lui, analyse la position de Louis Veuillot au sein même du romantisme français.

12. John Hare, Le Canadien et sa femme: une brocbure québécoise de propagande politique (1794), avec introduction et notes, Département de Lețres françaises, Université d'Ottawa, 1994, $30 \mathrm{p}$.

13. John Hare, Aux origines du parlementarisme québécois (1791-1793), Sillery, Le Septentrion, 1993, 312 p.

14. Maurice Lemire (dir), La Vie littéraire au Québec, tome 1 (1764-1805). La voix française des nouveaux sujets britanniques (1764-1805), Presses de l'Université Laval, 1991, p. 146-160.

15. Bernard Andrès (dir.), Principes du littéraire au Québec (1766-1815), Montréal, Université du Québec à Montréal, Département d'Études littéraires, Cahiers de l'ALAQ, no 2, août 1993, 66 p.

16. Voir Fortune et Infortunes d'un dandy canadien. Pierre-Jean de Sales Laterrière: Journal de voyage (1815), Montréal, Université du Québec à Montréal, Département d'études littéraires, Cahiers de l'ALAQ, no 3, hiver 1994, 92 p. (édition et présentation de Bernard Andrès et Pierre Lespérance). L'essai de Pierre-Jean de Sales Laterrière est paru sous le pseudonyme de A Canadian, sous le titre $A$ Political and Historical Account of Lower Canada; with Remarks in the Present Situation of the People as Regards Their Manners, Character, Religion, \&, \&, London, William Marsh And Alfred Miller, 1830, xvi-275 p.

17. Revue américaine de Seattle, États-Unis, à laquelle participeraient des chercheurs québécois.

18. Norbert Spehner, Écrits sur le roman d'espionnage. Bibliograpbie analytique et critique des études et essais sur le roman et le film d'espionnage, Québec, Nuit blanche, 1994.

19. Aurélien Boivin, Maurice Émond et Michel Lord (dir.), Les Ailleurs imaginaires. Les rapports entre le fantastique et la science-fiction, Nuit blanche, 1993, $308 \mathrm{p}$.

20. Michel Lord, «Problématique du fantastique et de la SF , ibid., p. 105.

21. Paul Bleton, Les Anges de Machiavel. Essai sur le roman d'espionnage, Québec, Nuit blanche, 1994, $360 \mathrm{p}$.

22. Sur ces questions, voir le livre de Saint-Jacques et de la Garde, Les Pratiques culturelles de grande consommation, Nuit blanche, 1992, signalé dans ma chronique du numéro 54, p. 643-644.

23. Souvent invoqué, l'esprit de Marc Angenot plane utilement sur ce travail de longue haleine. 